\title{
CURRICULUM Y DESCOLONIZACIÓN: LA CONSTRUCCIÓN DE UN CURRICULUM CRÍTICO EMERGENTE EN UNA ESCUELA BÁSICA COMUNITARIA MAPUCHE LAFQUENCHE, LLAGUEPULLI, REGIÓN DE LA ARAUCANÍA (CHILE)
}

\author{
CURRICULUM AND DECOLONIZATION: CONSTRUCTION OF AN EMERGING \\ CRITICAL CURRICULUM ON A COMMUNITARIAN MAPUCHE LAFQUENCHE \\ PRIMARY SHCOOL, LLAGUEPULLI, REGIÓN DE LA ARAUCANÍA (CHILE)
}

Rolando Pinto Contreras ${ }^{1}$

Doctor en Ciencias de la Educación

Pontificia Universidad Católica de Chile

Santiago

rolopintocontreras@gmail.com

\begin{abstract}
Resumen: En el contexto político conservador de la Educación Nacional de Chile, en el año 2010 surge el interés de algunas comunidades Mapuche Lafquenche de Llaguepulli, Región de La Araucanía (Chile) de afirmar su identidad cultural ancestral y elaborar para sus Escuelas Básicas Comunitarias un Curriculum Autóctono, que rescate los saberes y las prácticas ancestrales de vida del pueblo mapuche.

En esta experiencia constructiva, que duró 8 años (2011-2018), tuvimos la preocupación epistemológica y política de no reproducir la racionalidad técnica instrumental que hubiese significado continuar con una visión neocolonialista, que siempre ha tenido, al menos en Chile, la acción de diseñar y desarrollar currículos diferentes al oficial.

Con este artículo recogemos parte de esa experiencia y la reflexionamos como "aportes a una política curricular descolonizadora" que haga más pertinente la educación a las necesidades e intereses de liberación, en este caso, del pueblo Mapuche Lafquenche.
\end{abstract}

\footnotetext{
${ }^{1}$ Académico Chileno, Doctor en Ciencias de la Educación (Bélgica, 1979), Sociólogo del Desarrollo (Bélgica, 1976) y Profesor de Estado en Filosofía (Chile, 1968). Actualmente jubilado de la Pontificia Universidad Católica de Chile, vinculado como asesor Curricular con Comunidades Mapuche Lafquenche, Llaguepulli (Lago Budi) y con otras Comunidades Mapuches, ubicadas en diversas localidades de la Región de la Araucanía; y con el Programa de Maestría en "Educación de Jóvenes y de Adultos y procesos formativos", en la Universidad de Playa Ancha (Valparaíso). Además, desarrolla diferentes consultorías nacionales e internacionales sobre "Modelos Curriculares Innovadores para la Educación entre Adultos", netamente su propia propuesta del "Curriculum Crítico Emergente" y sobre "Extensionismo Rural Interactivo entre pares", procurando aplicar una metodología de educación popular crítica/emancipadora. Su email de contacto es: rolopintocontreras@gmail.com
} 
Palabras Claves: Educación Pública Descolonizada; Curriculum Crítico Emergente; Construcción Curricular interactiva e intercultural; Cultura ancestral Mapuche Lafquenche.

Abstract: In the conservative political context of the National Education of Chile, in 2010 the interest of some Mapuche Lafquenche communities of Llaguepulli, from Araucanía Region (Chile) arose to affirm their ancestral cultural identity and develop an Indigenous Curriculum for their Community Primary Schools, to rescue the ancestral knowledge and life practices of the Mapuche people.

In this constructive experience, which lasted 8 years (2011-2018), we had the epistemological and political concern not to reproduce the instrumental technical rationality that would have meant continuing with a neocolonialist vision, which has always had, at least in Chile, the action of design and develop curricula different from the official one.

With this article, we collect part of that experience and reflect it as "contributions to a decolonizing curricular policy" that makes education more relevant to the needs and interests of liberation, in this case, of the Mapuche Lafquenche people.

Key words: Decolonized Public Education; Emergent Critical Curriculum; Interactive and intercultural Curriculum Construction; Ancestral Mapuche Lafquenche culture

\section{1.- UNA BREVE INTRODUCCIÓN EXPLICATIVA}

A pesar de los avances que a nivel del reconocimiento internacional de los Derechos Humanos, Sociales y Culturales de los pueblos indígenas, a usar y conservar su lengua original y a que los sistemas educativos nacionales deben reconocer en su organización curricular áreas de saberes y prácticas ancestrales de las culturas indígenas, en Chile, país que ha suscrito la mayoría de los convenios internacionales pertinentes que norman sobres estos derechos, sólo en el año 1993 se dicta la Ley № 19.253 que junto con crear la Corporación Nacional de Desarrollo Indígena, en sus artículos 28 y 30 norman sobre el reconocimiento de que en las áreas de alta concentración poblacional indígena se introduzcan al Curriculum Oficial objetivos y contenidos de enseñanza de las lenguas tradicionales indígenas y algunas actividades que permitan conservar ciertas prácticas artesanales y culturales ancestrales.

Pero incluso esta restricción no se pone en práctica hasta el año 2009 mediante el Decreto $\mathrm{N}^{\circ}$ 280, del Ministerio de Educación Pública mediante el cual se autoriza que, en la Región de la Araucanía, se haga el esfuerzo de una Educación Intercultural Bilingüe, que considere la enseñanza del Mapudungun (lengua original del pueblo mapuche); la contratación de Educadores Tradicionales Mapuche que organicen talleres y cultiven especies nativas de consumo mapuche y se hagan ajustes curriculares en los sectores de aprendizaje vinculados al arte y la tecnología, incluyendo en sus Programas de Estudios componentes estéticos y artesanía mapuche. 
Es decir, en la visión política y educativa del Estado Nacional se reconocía el derecho social del pueblo Mapuche para su autonomía cultural relativa, pero en su implementación práctica sólo se aceptaba una autonomía educativa parcial, que debía subordinarse a la regulación curricular oficial, supervisada por el Estado Nacional.

Mientras la lucha soberana por la devolución de los territorios ancestrales del pueblo mapuche y la autonomía cultural plena seguían siendo los ejes centrales de sus luchas, la actitud y la fuerza del Estado Chileno persistían en la mantención de la política neocolonialista, también ancestral.

En este escenario de posiciones ancestrales antagónicas, en el año 2010 tomaron contacto con nosotros en la Universidad Metropolitana de Ciencias de la Educación/UMCE, en Santiago de $\mathrm{Chile}^{2}$, un grupo de autoridades políticas y educativas de una Comunidad Mapuche Lafquenche ${ }^{3}$, ubicada en la vecindad del Lago Budi, Comuna de Theodoro Smith, Región de la Araucanía (Chile), con el propósito de invitarnos a realizar un taller sobre "Diseño y Desarrollo Curricular para los directivos y docentes de la Escuela Básica Comunitaria KOM PU LOF NI KIMELTUWE"4.

Con ese taller ellos pretendían aprender a construir sus propios Planes y Programas de Estudios para las Escuelas Básicas Comunitarias que se ubican en el territorio Lafquenche de Llaguepulli.

Luego de conversar y analizar la poca viabilidad de que con ese taller surgiera algún diseño metodológico que les permitiera cumplir con su aspiración curricular, ya que tal construcción curricular era compleja y tenía varias etapas previas que desarrollar, antes de abocarse a esa construcción de Planes y Programas de Estudios propios, originales y autóctonos

Entre las acciones previas les advertimos del peligro neocolonialista que podría tener esta construcción curricular sin previamente no tener claro el sentido político emancipador de esa elaboración curricular; les señalamos, que una manera de evitar ese proceso neocolonialista era que ellos tuvieran sistematizados los saberes y prácticas ancestrales de la cultura Mapuche Lafquenche, que permitiera caracterizar los posibles sectores de

\footnotetext{
2 En ese año, desde Mayo de 2010, había asumido la Dirección de Postgrado, en la Universidad Metropolitana de Ciencias de la Educación/UMCE, en Santiago de Chile y en ese carácter recibí la visita del Lonco (Jefe Político de una Comunidad Mapuche), señor Jorge Calfuqueo y del Director de la Escuela Básica Comunitaria de Llaguepulli KOM PU LOF ÑI KIMELTUWE, señor Hugo Painequeo. En ese cargo estuve 02 años, renunciando en el mes de Octubre, 2012, por "perdida de la confianza política" del Rector de la UMCE.

${ }^{3}$ La doble nominación MAPUCHE LAFQUENCHE obedece a la ubicación territorial específica de la comunidad Mapuche, lo distintivo de la identidad territorial es el concepto LAFQUENCHE, que se refiere a la ubicación de la Comunidad en un territorio próximo a una geografía lacustre (cerca del mar, de lagos o de ríos). La etnia es siempre Mapuche (hombre o mujer de la tierra) y el territorio específico es el Lafquenche (hombre o mujer del agua).

${ }^{4}$ La traducción al castellano es: TERRITORIO ABIERTO PARA LA FORMACIÓN DE NIÑOS Y NIÑAS DEL PUEBLO MAPUCHE.
} 
aprendizaje autóctonos sobre los cuales se debieran elaborar esos Planes y Programas de Estudios propios que deseaban construir.

También como un factor co-ayudante de esa visión descolonizadora era importante que algunos historiadores mapuches y algún abogado mapuche, hicieran un estudio sobre alguna experiencia constructiva de una educación propia mapuche y sobre la viabilidad legal de que una comunidad relativamente pequeña tuviese la posibilidad de construir su propio modelo curricular, contra lo que establecía la legalidad indígena vigente.

Además, había que seleccionar a los académicos especialistas en curriculum que, junto con ellos y sus comunidades, participarían de este proceso. Esto significaba, entre otros aspectos, que tuviesen claridad epistemológica y política descolonizante, o al menos estuvieran atento a cualquier desviación metodológica o instrumental que se adoptara en esa construcción curricular.

Por último, estaban los costos humanos y financieros que suponía realizar este proyecto curricular.

Nuestros argumentos los centramos en que ellos entendieran la lógica y el tiempo que tomaría concretar su aspiración curricular, en un clima de respeto mutuo a nuestras respectivas cosmovisiones epistemológicas y políticas.

La aspiración de tener Planes y Programas de Estudios propios para la educación básica de los niños y las niñas Mapuche Lafquenche, era, en ese entonces, una propuesta política de autonomía cultural y de afirmación de la identidad popular comunitaria ancestral, pero no se comprendía la complejidad teórica, técnica y política de esa elaboración curricular.

Fue ahí que ambas autoridades, que ya conocían nuestra propuesta teórica sobre el Curriculum Crítico Emergente 5 , fuertemente motivados por la lectura del libro que habíamos publicado en la Editorial de la Universidad Católica de Chile (PUCH), en el año 2008, y que desarrollaba la alternativa de "construir" Curriculum Crítico Emergente, con la participación y el protagonismo de las personas y organizaciones comunitarias que sostenían dicha posibilidad constructivista, entendieron la complejidad de la acción y la amplitud de tiempo y de recursos que se requerirían para materializar su experiencia curricular autóctona e inédita. Se comprometieron a buscar las asesorías legales que les permitiera asumir su motivación política emancipadora de impulsar y dirigir esa construcción curricular en el territorio Mapuche Lafquenche, de Llaguepulli, según su propia soberanía y en los tiempos necesarios para esa elaboración.

Al mismo tiempo reconocieron que no habían realizado aún una investigación y una sistematización en profundidad de su cultura ancestral y que desconocían si existía en el

\footnotetext{
${ }^{5}$ Ambos personeros eran Profesores de Educación General Básica, el Lonco con la mención de Castellano y Comunicación y el Director con Mención en Matemáticas y estaban cursando el Magister en Curriculum e Interculturalidad, en la Universidad de la Frontera de Temuco y habían leído y analizado el libro de nuestra autoría El Curriculum Crítico. Una Pedagogía Transformativa para la Educación Latinoamericana, publicado por la Editorial UC, en el año 2008.
} 
territorio de la Araucanía alguna experiencia de construcción curricular o educativa como a la que ellos aspiraban, más bien, en los estudios y conversaciones que ellos como pueblo tenían, en sus reuniones o ampliados comunitarios, no se conversaba sobre la implementación curricular mapuche. Ellos aseguraban que tenían "principios pedagógicos mapuches" y estos estaban sistematizados en el Proyecto Educativo Institucional de cada Escuela Básica Comunitaria, pero tampoco habían sistematizados estos principios como prácticas pedagógicas escolarizadas.

Al término de estas conversaciones acordamos elaborar conjuntamente un Proyecto de Intervención Curricular Crítica Emergente y buscar alguna fuente nacional o internacional que pudiera financiarlo por los años que demoraría esa elaboración. En el taller de Enero de 2011 elaboramos ese Proyecto y lo enviamos a dos fuentes externas de financiamiento: a la UNESCO/IESALC, con sede en Caracas y a Entraide et Fraternité, en Bélgica. En Marzo de 2011 firmamos el contrato con el primero de estos organismos, que nos financiaba por 2 años.

El Proyecto comprendió las siguientes acciones:

1) Investigar y sistematizar los saberes y las prácticas ancestrales, que conformaban la cultura Mapuche Lafquenche, en Chile y que serían la base cognitiva y el sentido curricular emergente sobre la cual deberían elaborarse los Planes y Programas de Estudios propios. Esta acción significaba investigar en textos disponibles y en entrevistas interactivas con diversas autoridades ancestrales mapuche, sobra la visión y los ejes constitutivos de la Cultura Mapuche Lafquenche.

2) Luego había que sistematizar esos saberes y prácticas ancestrales y organizarlos en una Matriz Curricular Autóctona y original, que describiera esos saberes y prácticas, como Ámbitos o Sectores o Asignaturas de Aprendizaje de lo autóctono; definir principios y estrategias pedagógicas por ámbito, que provinieran de la tradición educativa mapuche y un conjunto de orientaciones pedagógicas sobre la organización curricular en la Escuela (horas lectivas y trabajo en el hogar de los/las estudiantes; material pedagógico de apoyo para los/las docentes y para los/las educandos/as; indicadores y formas de evaluación de aprendizaje; y gestión del nuevo currículo en la Escuela).

3) En tercer lugar había que elaborar los Planes y Programas de Estudios propios por cada uno de los ámbitos de aprendizaje de lo autóctono, ordenados en una gradualidad progresiva del aprendizaje, según la crianza familiar ancestral mapuche $\mathrm{y}$ adoptar/adaptar las disposiciones y formularios oficiales del Ministerio de Educación, compatibilizando los Ciclos de Formación de las Escuelas Básicas Comunitarias de Llaguepulli, con la división de grados escolares básicos equivalentes, en que se organiza la educación nacional.

Estas 03 acciones descritas, con optimismo, podrían tomar entre 03 a 04 años de elaboración curricular.

4) Finalmente, toda esta elaboración curricular emergente debía ser presentada para su revisión institucional y para la aprobación legal ante el Consejo Regional de Educación, si la comunidad quería mantener los subsidios educacionales que 
reconoce la legislación chilena a las Escuelas Básicas Particular Subvencionadas del país.

Pues bien, los factores facilitadores del proyecto se fueron resolviendo rápidamente: la UNESCO/IESALC decide apoyar las dos primeras acciones y que culminaría con la caracterización y edición de la Matriz Curricular Autóctona; también desde el punto de vista legal los abogados asesores Mapuches habían encontrado en la ambigüedad de la Ley chilena y en los Convenios Internacionales reconocidos y asumidos por el Estado Nacional, los apoyos normativos que respaldaban esta elaboración curricular; y desde el punto de vista de los recursos humanos técnico-políticos y de las comunidades mapuche Lafquenche, nos comprometíamos para la continuidad de las acciones previstas en el proyecto.

La ejecución del proyecto tuvo entonces tres etapas de construcción curricular: una primera que consistió en la investigación/sistematización de los saberes y las prácticas ancestrales Mapuche Lafquenche y la elaboración con ese material de la Matriz Curricular para las Escuelas Básicas Comunitarias de Llaguepulli; una segunda etapa, la más larga del proceso curricular, la elaboración de Planes y Programas de Estudios propios para cada uno de los ámbitos socio-culturales ancestrales (en total se caracterizaron 08 ámbitos o sectores de aprendizaje autóctonos) y la adecuación/contextualización de las 04 Asignaturas Mínimas Obligatorias de la Educación Básica Nacional (Matemáticas; Castellano y Comunicación; Ciencias Naturales y Ciencias Sociales) que se debían adecuar a los principios formativos en la cultura y la tradición Mapuche; y una tercera etapa, la revisión técnica política del Ministerio de Educación Nacional sobre la propuesta de Planes y Programas de Estudios propios de dos escuelas básicas comunitarias de Llaguepulli: la KOM PU LOF ÑI KIMELTUWE, de Llaguepulli bajo y la KIMELTUCE MAPU, de la comunidad de Llaguepulli alto; sin embargo la Secretaria Regional de Educación definió que solo se haría esta construcción curricular, de manera piloto, en la Escuela KOM PU LOF. La discusión de las observaciones técnicas y políticas sobre esa propuesta, que señalaran los expertos curriculares interculturales del Ministerio y, finalmente la aprobación legal de la propuesta piloto del Curriculum Emergente Crítica, para la Escuela KOM PU LOF ÑI KIMELTUWE, de parte del Consejo Nacional y Regional de Educación.

La primera etapa (2011-2012) contó, como decíamos, con el apoyo económico de UNESCO IESALC. La segunda etapa a partir del mes de Agosto de 2012 (2012-2016), tuvo diversas fuentes de financiamiento internas de Chile (CORFO Innovaciones y CONICYT) y del Extranjero, principalmente de Organizaciones de Pueblos Originarios de Canadá y Nueva Zelanda, recursos propios de los académicos participantes y el soporte permanente de las Comunidad Mapuche Lafquenche de Llaguepulli; y la tercera etapa "Revisión técnica-política de la propuesta de Planes y Programas propios para la Escuela Básica Comunitaria de Llaguepulli", hasta su aprobación legal, fue financiada por el Proyecto de Educación Intercultural Bilingüe, de la Secretaría Regional de Educación de la Araucanía.

Así se realizó el proyecto que lo diseñamos interactivamente en el Taller de Enero de 2011 y que culminó en Marzo de 2018, con la autorización legal del Consejo Regional de Educación para que la Escuela Básica Comunitaria KOM PU LOF ÑI KIMELTUWE comenzara 
a aplicar en el Ciclo Inicial de la escolaridad de esta escuela (Pre-básica, 1er y 2do grados escolares) los nuevos Planes y Programas de Estudios propios.

En lo que sigue de este texto queremos mostrar la doble experiencia constructiva que nos desafió; por un lado, aprender a dialogar entre dos visiones epistemológicas y educativas, con peso de significaciones totalmente diferentes, incluso con el dominio conceptual lingüístico absolutamente distinto, pero que para los efectos de la construcción curricular eran equivalentes y complementarios: la cosmovisión ancestral Mapuche y la Cosmovisión Humanista Critica Emancipadora que compartíamos los/las académicos/as que participábamos y apoyábamos esta experiencia curricular.

Y, por otro lado, constituir una comunidad de aprendizaje soberana que, basado en el respeto mutuo, y en la construcción interactiva de un modelo curricular crítico emergente inédito y fundacional para la cultura y la educación mapuche en el país, estuviera atento a cualquier desviación o manipulación ideológica que distorsionara el sentido emancipador y descolonizador de este trabajo.

Desde el punto de vista formal de la elaboración de este texto, tomaremos como apartados del mismo cada una de las acciones que estaban señaladas en el Proyecto inicial del año 2011, remarcando en ellas el sentido comunitario y colaborativo de esta construcción, así como su carácter soberano, autónomo y original que nos aseguraba nuestra perspectiva anti neocolonial. Por lo mismo, en un apartado final de este texto resaltamos el carácter emancipador y no colonialista de eta experiencia de construcción de un Curriculum Crítico Emergente, en las Escuelas Básicas Comunitarias de Llaguepulli.

\section{2.- LAS ACCIONES DE CONSTRUCCIÓN INTERACTIVA E INTERCULTURAL DEL CURRICULUM CRÌTICO EMERGENTE PARA LA ESCUELA BÁSICA COMUNITARIA DE LLAGUEPULLI.}

\section{1.- La Recuperación y Sistematización de los Saberes y las Prácticas Ancestrales Mapuche Lafquenche.}

Las principales fuentes informativas fueron: los pocos textos de historias y de relatos mapuches disponibles en el Centro de Documentación de la Araucanía, ubicado en Temuco; las investigaciones que habían realizado algunos Centros de Antropología de algunas Universidades Regionales, de la Araucanía, principalmente la Universidad de la Frontera y la Universidad Católica de Temuco; y las conversaciones y relatos directos con el Lonco (Jefe Político de la Comunidad), el Machi (Jefe Espiritual y de la Sanación Comunitaria), el Werken o representante legal de la Comunidad de Llaguepulli; el Director de la Escuela y las diversas personas con funciones distintivas de la Comunidad: la "Papay", mujer sabia de la tradición oral histórica de la comunidad; la Encargada Culinaria de la Comunidad, que tiene su propio restaurante étnico, con un profundo conocimiento sobre la calidad nutritiva de los productos naturales y de los cultivos tradicionales mapuche Lafquenche; la Encargada de la Huerta de Plantas Medicinales autóctonas; todos los demás integrantes encargados de diversos 
proyectos de desarrollo de la tradición comunitaria y los/las docentes de la Escuela Básica Comunitaria.

El equipo encargado de hacer esta investigación interactiva fuimos el Lonco y el Director de la Escuela, 02 Académicos especialistas en Curriculum de la UMCE; 01 Académico especialista en Geografía Humana también de la UMCE y 01 Académico especialista en Antropología y Etna-lingüista, de la Universidad de Concepción.

Como estrategia metodológica para este rescate y sistematización cultural, consensuamos adoptar una estrategia de Investigación Acción Participativa, procurando siempre que etnográficamente predominara la textualidad del relato de las autoridades comunitarias y de sus docentes Mapuche. Este respeto etnográfico es lo que le dio la legitimidad sociocultural y comunitaria a este proceso de sistematización de la cultura ancestral Mapuche.

Las reuniones de trabajo de este equipo funcionaban como comunidades de aprendizaje entre adultos en que particularmente los académicos involucrados éramos los sujetos en formación y el Lonco y el Director, los educadores situados.

Después de recoger toda esta información escrita y oral, organizamos la misma según saberes y prácticas ancestrales y consuetudinarias que englobaban la visión cultural mapuche; este ordenamiento nos permitió definir y caracterizar los 08 Ámbitos Centrales de la cultura Mapuche Lafquenche, que constituían el contenido fundamental de la MATRIZ CURRICULAR para la Escuela KOM PU LOF ÑI KIMELTUWE.

Sin embargo, en el desarrollo de esta primera acción ya connotábamos la construcción interactiva de una Comunidad Investigativa y de Aprendizaje, entre aquellos que entregaban la información y aquellos que al comprenderla y analizarla íbamos orientando la sistematización conjunta de los saberes y las prácticas ancestrales Mapuche Lafquenche.

\section{2.- La Matriz Curricular de la Escuela Básica Comunitaria Kom Pu Lof Ñi Kimeltuwe.}

El mismo equipo investigador, ampliado a los/las docentes de la escuela básica comunitaria, elaboró la Matriz Curricular, entendiéndola como el documento base del proyecto de construcción curricular. En ella se define:

- El sentido político y cultural de la comunidad de Llaguepulli a tener un Currículo y una Escuela autóctono/a; el derecho que les reconoce la Ley Indígena Chilena, redefinida en el año 1995, de hablar su legua, el MAPUDUNGUN; vivir en paz en sus territorios, afirmando sus valores comunitarios, en armonía y equilibrio vital con la Naturaleza y la Biodiversidad Natural.

Pero donde también se cruzaba la reivindicación histórica mapuche: la devolución de los territorios nativos, ocupados hasta ese entonces por la Misión Religiosa y Educativa de la Araucanía de la orden religiosa de los Capuchinos. Esta devolución había comenzado a ser realidad en el año 2005, cuando los Capuchinos entregan las tierras y las instalaciones, entre otra la Escuela Básica, pero sólo culmina en el año 
2015 cuando finalmente se hace el traspaso legal de esos bienes a la Comunidad y los curas Capuchinos abandonan la región, regresando a Alemania su patria imperial. ${ }^{6}$

- La Matriz en sí tiene una introducción/fundamentación que corresponde al punto anterior y luego 04 Columnas: en la primera se describen/definen los 08 ámbitos de saberes y prácticas ancestrales mapuche Lafquenche; en la segunda columna se define el sentido pedagógico mapuche que debía orientar la enseñanza y el aprendizaje de los contenidos que se derivaban de estos 08 ámbitos, según la tradición de la crianza y la formación de los niños y las niñas mapuche en su vida familiar, lo que debía quedar explícito en la matriz que desarrollaba el Plan de Estudio de ese ámbito; en la tercera columna se orientan los énfasis pedagógicos diversos que asumen las actividades de enseñanza y aprendizaje que correspondían a la progresión del desarrollo integral de los niños y las niñas, hasta lograr en su egreso de la escuela básica comunitaria los rasgos culturales e integrales del ser mapuche Lafquenche y en la cuarta columna se señalan las características de la evaluación e indicadores de logro que deben alcanzar los/las educandos al término de su formación básica.

- Los 08 ámbitos del aprendizaje ancestral Mapuche Lafquenche son:

a) AMULEPE TAIÑ MOGEN (Historia y cultura Mapuche). Corresponde a los relatos de la tradición oral que reconstruyen la existencia y las vivencias de todo tipo del pueblo Mapuche, desde la llegada de los españoles y hasta su evolución actual. Se trata de relaciones de opresión, en que el Estado Chileno y las clases usurpadoras del territorio Mapuche, han ido sistemáticamente destruyendo los símbolos identitarios de la cultura autóctona Mapuche.

b) AWKANTUN EGU AYEKAN (Deporte, juegos y recreación). El juego y la música es la unión del LOF, es la amistad y reciprocidad que explica la relación armoniosa del CHE con la naturaleza y entre los/las Mapuche.

c) AZ MOGEN WAJONTU MAPU MEW (Cosmovisión del tiempo y el espacio). Este ámbito se refiere a la comprensión y a la práctica vital entre el ser Mapuche con los diversos espacios territoriales y con los seres tangibles e intangibles que conviven en el LOF. Y esto no sólo como una vivencia pasada, sino que actual de compartir en el LOF: familia, territorio e historia cultural Mapuche.

d) IXOFIJ MOGEN (Biodiversidad). Se agrupan aquí los saberes y las prácticas de convivencia cotidiana con la diversidad de seres tangibles e intangibles que pueblan la Naturaleza próxima territorial. Se trata de respetar ese equilibrio cósmico de la vida natural y de las relaciones de armonía y reciprocidad que estable el Mapuche con su territorio natural y comunitario.

e) KUME MOGEN (Salud, Nutrición y Sanamiento). Se parte de la visión de armonía y equilibrio vital del hombre y la mujer Mapuche. Para ellos debe saber alimentarse y cultivar los productos ancestrales que los nutren; reconocer las características

\footnotetext{
${ }^{6}$ La historia de la Misión de la Araucanía que sustento la colonización de las tierras mapuche en todas las provincias del Sur de Chile ha sido documentada en varias publicaciones académicas y de intelectuales e historiadores Mapuche; entre otras la Escucha Huinca; La usurpación Alemana en el Sur de Chile; la historia de la dominación religiosa en la Araucanía; etc.
} 
de las plantas medicinales y de su cultivo; en fin, siempre saber llevar una vida saludable y equilibrada.

f) MAPUCHE FEYENTU (Espiritualidad Mapuche). En la práctica de las Ceremonias Comunitarias de Espiritualidad, el hombre y la mujer Mapuche se comunica con los diversos GHE y mediante el/la MACHI dialoga con cada uno de ellos. En los espacios naturales del territorio circulan estos GHE y en la noche ellos recuperan su energía vital que se la transmiten en el día a su pueblo. Se trata de un equilibrio cósmico que siempre está presente en la vida comunitaria Mapuche Lafquenche.

g) RAKIZUAM KA MAPUDUNGUN (Pensamiento y Lenguaje). Se trata de conocer el habla de la naturaleza e imitando su expresión audífona se va constituyendo en la lengua MAPUDUNGUN. Por eso la lengua Mapuche tiene un sentido de identidad personal y sociocultural ancestral. Su práctica comunicativa cotidiana desarrolla el pensamiento lógico del ser Mapuche y la capacidad creativa de nombrar la realidad.

h) ZOMO KUZAW KA KUZAW WENXU (Trabajo Manual y Artesanía Mapuche). Se trata de la valoración y del aprender a hacer manualidades, que tienen símbolos, formas, coloridos y contenidos propios de la tradición cultural y de la vida doméstica Mapuche. En general se trata de actividades que concilian el arte, la vivencia doméstica familiar y la tradición oral del aprendizaje artesanal Mapuche. Tradicionalmente el arte y la artesanía mapuche utiliza 04 materiales diversos y que son consuetudinarios en la cultura mapuche y que están disponibles en el Ixofij Mogen del territorio Lafquenche: Ia lana de oveja (que es la base de la elaboración de prendas para el abrigo humano); la madera y las fibras vegetales (que es la base de elaboración de utensilios domésticos: fuentes, instrumentos de labranza, canastos y bandejas tejidas); la greda o tierra arcillosa (base de la elaboración de utensilios para la elaboración de alimentos y depósitos de líquidos); y la plata, el cobre, piedras de colores u otros minerales disponibles o accesibles en el mercado local próximo (principalmente para la elaboración de joyas y utensilios para comer).

- Por último, esta Matriz orienta la elaboración de los Planes y Programas de cada ámbito de aprendizaje y para cada Ciclo de formación que organizaba a la Escuela Básica Comunitaria. Esta escuela está organizada en torno a 04 Ciclos de Aprendizaje: Inicial (Pre-básico, 1o y 2o básicos); Intermedio Bajo (3ero y 4to básicos); Intermedio Alto (5to y 6to básicos) y de Profundización (7mo y 8avo básicos).

Para los tres primeros Ciclos, cada uno es atendido por un/una docente mapuche y un/una docente colaborador/ra, también mapuche; y para el Ciclo de Profundización que es atendido por un/una docente que es apoyado/a por los/las demás docentes que tengan mención en las 04 asignaturas obligatorias mínimas del Plan Nacional Oficial.

Todas estas características de la Matriz la transformaron en la carta de navegación para nuestra continuidad del trabajo de construir el Curriculum de cada ámbito de saberes y prácticas ancestrales Mapuche Lafquenche. 
Según el corolario de esa Matriz Curricular, la que era explicada por el Lonco y por el Director de la Escuela KOM PU LOF ÑI KIMELTUWE, como un primer e histórico producto curricular mapuche elaborado colaborativamente por los docentes de la Escuela; los miembros de la Comisión de Educación de la Escuela ${ }^{7}$ y los académicos que apoyábamos esa elaboración. La Matriz era reconocida como un producto comunitario educacional y en cuanto tal, una guía curricular que debía orientar toda la educación que impartiera la Escuela.

Aquí es importante destacar que la Matriz es visualizada como una guía doctrinaria de la construcción curricular comunitaria mapuche y no como un instrumento técnicoinstrumental funcional sólo para elaborar Planes y Programas de Estudio.

Sin embargo, para la elaboración de esta Matriz se siguió funcionando como una Comunidad de Aprendizaje donde se iban alternando los roles de docente/aprendiz según el componente de elaboración de la columna que la constituía; así en las dos primeras columnas fueron los docentes de la Escuela los que asumieron el rol de enseñantes y en los columnas tres y cuatros fuimos los académicos participantes, los que protagonizamos su elaboración. Pero siempre funcionando como una comunidad de aprendizaje entre educador/educando y Educando/Educador.

\section{3.- La Elaboración de los Planes y Programas de Estudios Propios.}

Para la elaboración de cada Plan de Estudio por Ciclo de Escolarización y de los Programas que se derivaban de ese Plan, por ámbito autóctono o sector nuevo de Aprendizaje, se definieron los equipos responsables para cada ámbito nuevo. Estos equipos estaban constituidos por un/una docente de la Escuela Básica Comunitaria, que asumía la coordinación del trabajo curricular; los estudiantes de pedagogía y antropología en proceso de elaboración de sus trabajos finales de graduación y que asumían la tarea de llenar los formularios correspondientes normados por el Ministerio de Educación y; como asesor técnico político de la elaboración curricular, alguno de los académicos involucrados en el Proyecto o el Lonco o Director de la Escuela Comunitaria.

Lo importante de estos equipos es que funcionaron como comunidades de aprendizaje en que todos re-formulaban su identidad de educadores: los docentes de la Escuela afirmándose como profesionales del currículo y los estudiantes de pedagogía y de antropología asumiéndose como educadores/curriculistas situados. Pero todos/as como educadores/educandos del curriculum.

El diseño de Plan de Estudio que se usó en esta elaboración fue el establecido por el Ministerio de Educación para definir los Planes de Estudios Oficiales y desde el punto de vista

\footnotetext{
${ }^{7}$ La Comisión de Educación de la Escuela Básica Comunitaria KOM PU LOF ÑI KIMELTUWE fue creada en el año 1995, cuando la Comunidad de Llaguepulli se hace cargo de su gestión y se genera como instancia de asegurar la identidad cultural de la escuela y como representación de la autonomía comunitaria mapuche frente al Ministerio de Educación Nacional. Su constitución actual es un Presidente/a designado/a por la Asamblea Comunitaria, 04 miembros elegidos por funciones específicas por la Asamblea de Padres y Apoderados de la Escuela, el Director de la Escuela, un representante de los/las docentes y 02 representantes de los exalumnos/as.
} 
metodológico, adecuamos nuestra visión estratégica de investigación acción a las orientaciones interactivas de la Antropología Aplicada Interactiva del Sur, generada en la Escuela de Antropología de la Universidad Católica de Temuco. Esta adecuación metodológica significó que las reuniones de cada equipo se instalaban como conversaciones y reflexiones en que cada uno de los participantes era aprendiz y protagonista de ese ámbito de aprendizaje ancestral.

Según este refuerzo metodológico, los planes y programas de estudios en construcción debían tener como protagonistas a los docentes y directivos de la Escuela Básica Comunitaria y los estudiantes de pedagogía y de antropología eran ayudantes de los equipos docentes y re-aprendices de su perfil profesional en formación.

Por tanto, la matriz que se utilizó para el diseño de cada Plan de Estudio, adopto el carácter de un "Plan de Orientaciones Pedagógicas Generales" diferenciadas para cada Ámbito y para cada Ciclo de Formación; y de acuerdo a una carga de horas lectivas presenciales y de trabajo en casa de los/las estudiantes.

A su vez, los "Programas de Estudios por Ciclos Escolares", los detallamos en su estructura formal según las disposiciones legales que exigía el Ministerio de Educación.

En ambos procesos se involucraron voluntarios mapuches de la Comunidad, que les interesaba colaborar en la construcción de sus propios planes y programas de estudios, proceso que les permitía a su vez actualizar los conocimientos ancestrales y el sentido comunitario mapuche de las prácticas consuetudinarias.

Por su lado, los estudiantes de pedagogía y de antropología que estaban terminando sus estudios académicos de pregrado, y los asesores curriculares no mapuche (académicos de la UMCE y de la Universidad de Concepción) aprendíamos los sentidos holísticos de la cultura mapuche y situábamos nuestro conocimiento técnico científico en un contexto de realidad que nos provocaba en nuestro desempeño académico y profesional, en los espacios institucionales a los que pertenecíamos.

En este largo proceso interactivo de construcción curricular se fueron sumando otros académicos chilenos de otras Universidades Regionales, ubicados en Temuco, Valdivia y Villarrica, principalmente. Sobre todos como especialistas de un ámbito autóctono que nos ayudaban a comprender la precisión conceptual en MAPUDUNGUN y su equivalente en la expresión científica del Castellano.

Estos equipos interactivos e interétnicos fueron los que elaboraron el Curriculum Crítico Emergente de Llaguepulli8; fueron estos grupos integrados los que realizaban la

\footnotetext{
${ }^{8}$ La definición teórica de Curriculum Crítico Emergente que propusimos en nuestro libro se basaba en el conocimiento general que habíamos aprendido de diversas experiencias de educación popular en las que habíamos trabajo o evaluado, durante los años anteriores al 2008. En el Capítulo IV de nuestros libro "La mirada de futuro para la pedagogía y el currículo crítico de la educación latinoamericana" más que una reducción conceptual acotada, señala características en el propósito de diseño y en la construcción del currículo; así precisamos 03 características principales: su multiculturalidad; su inclusividad holística y su territorialidad
} 
investigación socio-cultural de los contenidos de cada ámbito de saberes y prácticas ancestrales Mapuche Lafquenche y los que iban organizándolos por Ciclos de Formación, dándole la gradualidad y la progresión del aprendizaje que los niñas y las niñas mapuche, traían desde la tradición familiar formativa mapuche.

La investigación interactiva recogió y sistematizó las textualidades relatadas, definidas y registradas en la memoria oral de las autoridades comunitarias y de cada miembro de esa comunidad, para cada ámbito de saberes y prácticas ancestrales y de la vida en el mundo natural y comunitario del territorio Lafquenche. Una vez sistematizadas las textualidades mapuche se procedió a transcribirlas a los formularios de Programas de Estudios normados por el Ministerio de Educación, pero en este caso para crear los Currículos Emergentes para cada ámbito cultural mapuche.

Tal como señalamos anteriormente, los Planes de Estudios se organizaron como Matrices de Orientaciones Pedagógicas Generales para cada ámbito de aprendizaje nuevo.

Estas “Orientaciones Generales” tienen un triple propósito:

- En primer lugar fijar la intencionalidad y las características que deben asumir los Programas de Estudios para cada ámbito de aprendizaje y para cada Ciclo de Formación;

- En segundo lugar ayudar a los/las docentes que asumen la tarea de organizar en el aula los Programas de Estudio, para la formación que deben impartir y los énfasis que deben colocar en ese ámbito y en ese Ciclo; y

- En tercer lugar, en el supuesto que se incorporen nuevos/as docentes mapuche a la escuela, que no participaron de esta construcción curricular, la lectura y análisis que haga de estas Orientaciones Generales y de los Programas de Estudios específicos, le permitirán auto-instruirse en la propuesta curricular propia.

Para esto la Matriz se organizó en 04 Columnas y un encabezamiento con el nombre del ámbito de aprendizaje y el Ciclo de formación al que se destinaba el aprendizaje. Las columnas identificaban los Objetivos Generales del Plan de Estudio (Primera Columna); Principales temas o contenidos que debían desarrollarse en cada uno de los Ciclos formativos (Segunda Columna); Orientaciones Didácticas Generales por Ciclo (Tercera Columna) y en la Cuarta Columna. "Principales Indicadores de Evaluación".

socio-cultural. Y con respecto a la construcción participación de los actores sociales que definen el currículo como proceso de construcción interactiva y la autonomía cultural de sus constructores. Lo interesante de la experiencia de Llaguepulli es que comprobamos en la práctica constructiva las 03 características teóricas y agregamos una nueva, lo paradigmático del sentido curricular ancestral y para el proceso de construcción la importancia del trabajo interdisciplinario. 
Estas Orientaciones Generales que se señalaban para cada uno de los 08 ámbitos autóctonos Mapuche Lafquenche, se diseñaron gradual y progresivamente para cada uno de los Ciclos de Formación en que se organizaba la Escuela KOM PU LOF ÑI KIMELTUWE.

En relación a los Programas de Estudios para cada ámbito y Ciclo de Aprendizaje, se adoptó el tipo de presentación formal de los Programas de Estudios Oficiales que usa el Ministerio de Educación en Chile. Así cada Programa quedó estructurado de la siguiente manera:

a) Nombre del Programa en el ámbito de aprendizaje específico y por Ciclo de Formación, en forma Bilingüe.

b) Propósito del Programa que fija la intencionalidad que se le da a ese ámbito y para ese Ciclo.

c) Nociones Básicas y principales contenidos temáticos del Programa para ese Ciclo. Estos contenidos se definen en MAPUDUNGUN.

d) Unidades de Aprendizaje por Semestre. Cada Unidad tiene los siguientes componentes: nombre temático de la Unidad, Objetivos de Aprendizaje con sus respectivos contenidos en Mapudungun, Indicadores de Evaluación de cada objetivo y Orientaciones Didácticas para el tratamiento de la Unidad de Aprendizaje en el aula. Se procuró describir 02 Unidades de Aprendizaje por Semestre y cada Unidad subdividida en 04 Objetivos de Aprendizaje, que tiene su correlato en los indicadores de evaluación?.

Los objetivos de aprendizaje se definían según la lógica de progresión inclusiva del niño o la niña mapuche a su vida familiar y comunitaria, por tanto, desde la mirada integral del aprendizaje cada objetivo apuntaba diferencialmente al logro valórico, cognitivo y del saber hacer de cada estudiante.

e) Además cada Unidad de Aprendizaje tiene un Objetivo de Aprendizaje Transversal que pone el acento a los aspectos valóricos y actitudinales que deben lograr los/las estudiantes mapuche de ese Ciclo Escolar.

f) También se hace una estimación de horas presenciales de clase semanal que va a ocuparse para el desarrollo de esa Unidad y los énfasis metodológicos/didácticos y actividades de aprendizaje que se desarrollarán en esa Unidad de Aprendizaje.

g) Cada Programa de Estudia explicita los indicadores y las estrategias de logros de aprendizaje integral que deben alcanzar los/las educandos/as en esa Unidad de

\footnotetext{
${ }^{9}$ En la cultura mapuche se trabaja con el $\mathrm{N}^{\circ} 4$ como base ordenadora, asimilando este número a lo que es el orden cósmico natural de la vida mapuche $(04$ orientaciones espaciales, 04 estaciones climáticas, y 04 los espíritus que rigen toda la vida mapuche) y sus múltiplos superiores e inferiores $(08,12$ y 02$)$. De ahí que el № de Unidades de Aprendizaje; los objetivos de aprendizaje y los ámbitos de saberes y prácticas ancestrales tienen esa visión cabalística proveniente de la cultura ancestral y consuetudinaria mapuche.
} 
Aprendizaje; para ello los objetivos de la Unidad deben facilitar la determinación de indicadores específicos para cada Unidad de Aprendizaje.

Una vez que el grupo de trabajo terminaba la elaboración del Plan y de los Programas de Estudios para cada ámbito de aprendizaje, se presentaban a la Asamblea de la Comunidad Educativa de la Escuela KOM PU LOF ÑI KIMELTUWE y ahí se corregían y se aprobaban las nuevas propuestas curriculares. Se trataba de Asambleas dirigidas por el Presidente de la Comisión de Educación y por el Director de la Escuela y de viva voz se señalaban las correcciones que había que realizar en esa propuesta borrador. Para asegurar estas correcciones se nombraba una Comisión Redactora con docentes y miembros de la Comunidad, donde los estudiantes y los académicos quedábamos fuera.

Toda esta construcción curricular se terminó de elaborar en Mayo de 2016 y el Documento con la propuesta de Planes y Programas de Estudios propios se entregó a la Dirección Regional de la Araucanía, en Setiembre de ese mismo año.

\section{4.- La Aprobación Legal de los Planes y Programas de Estudio Propios para las Escuelas Básicas Comunitarias de Llaguepulli.}

Uno de los procesos más demorosos, contradictorios y complejos que nos tocó vivir y en muchos momentos observar perpléjicamente, fue este proceso de discusión entre los actores educacionales Mapuche Lafquenche y los técnicos y especialistas en Curriculum "Intercultural", del Ministerio de Educación. Presenciamos en vivo y en directo la confrontación de dos racionalidades opuestas de concebir la Educación y el Curriculum.

Muchas veces nos tocó intermediar en la discusión, solicitando que los negociadores interactuaran tratando de entenderse en un propósito común: tener una educación de más calidad para los niños y las niñas mapuche Lafquenche y de reconocimiento y respeto a la autonomía mapuche de la Comunidad Lafquenche de Llaguepulli.

El documento que centró esta larga discusión fue un "Informe Técnico Curricular" elaborado por una Comisión de la Secretaría de Educación Regional y con expertos curriculares del Ministerio Central (en total 08 "especialistas", algunos de ellos mapuche) que objetaban aspectos de corrección formal y hasta visiones técnico-políticas de la propuesta Mapuche Lafquenche. En total eran 58 observaciones imperativas, es decir, que debían ser realizadas en la nueva versión de la propuesta. De estas observaciones la comunidad de Llaguepulli aceptó sólo 12: 04 de formas (aplicar de manera más normativa los instrumentos de Planes de Estudio y de descripción de los Programas propios); otras 04 de adecuación de la propuesta a las exigencias de la Normativa del Curriculum Oficial (por ejemplo, la inclusión de alguna taxonomía de los objetivos de aprendizaje, que centrara la progresión de logros y la evaluación de resultados) y 04 de fondo pero que solicitaban discutirlas, todas 
relacionadas con la aplicación del MAPUDUNGUN o del MAPUZUGUN en la nominación de los ámbitos de saberes ancestrales ${ }^{10}$ ).

Después de 01 año y medio de discusiones, el Consejo Regional de Educación Nacional, logró aprobar la propuesta curricular de la Comunidad Mapuche Lafquenche de Llaguepulli, los que están siendo aplicados en la Escuela Básica Comunitaria KOM PU LOF ÑI KIMELTUWE, de Llaguepulli, desde Marzo de 2018.

\section{3.- ALGUNAS REFLEXIONES FINALES, PERO AÚN EN PROCESO.}

Siempre nos llamó la atención la facilidad con que conseguimos hacer una construcción curricular crítica emergente para la Escuela Básica Comunitaria KOM PU LOF ÑI KIMELTUWE, de manera interactiva y funcionando como una Comunidad de Aprendizaje intercultural; ello, a nuestro juicio, se debe a tres factores colaborativos que estaban instalados en las prácticas comunitarias y en los académicos que participamos en dicha construcción.

En este sentido, estos factores garantizaban una perspectiva descolonizadora en la construcción curricular. Estos factores son:

\section{1.- La Pre-Existencia de una Concepción Originaria y Autónoma del Aprendizaje y su Progresión Pedagógica.}

"Nosotros tenemos ciertos principios educativos tradicionales que determinan líneas de procesos progresivos de formación para cada Ciclo del Desarrollo Humano Comunitario. Los/las niños y niñas que asisten a la Escuela muestran lo que ya aprendieron en sus familias y luego progresan. De esta manera de la misma forma en que ellos/ellas van asumiendo cognitiva, afectiva, valórica y activamente esos saberes y prácticas ancestrales de su vida comunitaria, en la Escuela se van sistematizando y desarrollando progresivamente los principios pedagógicos que los/las van constituyendo en su ser mapuce y en su identidad Lafquenche" (Hugo Painequeo, Taller/Seminario de elaboración del Proyecto "Construcción del Curriculum Crítico Emergente para la Escuela KOM PU LOF ÑI KIMELTUWE, de Llaguepulli; Enero de 2011).

Estos principios que desde la familia y hasta la vida plena comunitaria de los niños y las niñas Mapuche, son:

- Desarrollar el "Rakizuam" desde la progresión del aprendizaje del MAPUDUNGUN y hasta la construcción del pensamientos identitario Mapuche Lafquenche.

\footnotetext{
${ }^{10}$ El MAPUDUNGUN es la expresión general de la lengua Mapuche, pero hay nominaciones o énfasis lingüísticos del MAPUDUNGUN que adquieren connotaciones territoriales distintas y esto es reivindicado por las comunidades Lafquenche, que prefieren hablar de MAPUZUGUN.
} 
- Desarrollar el Kimvn" que progresa desde el reconocimiento intuitivo de los saberes y las prácticas ancestrales de identidad Mapuche Lafquenche y hasta la comprensión y el compromiso con la preservación y desarrollo de los diversos "Kimvn" existentes en la comunidad, tanto aquellos que tienen que ver con la esencia del ser mapuce, como en aquellos que se aplican cotidianamente en la vida familiar y comunitaria.

- Desarrollar el "Kimkantvn" que progresa desde el reconocimiento táctil los diversos "pu Kuzaw"; las actividades, vestimentas y alimentos propios de su cultura; a los diversos seres vivos, nativos, domésticos y naturales del entorno y hasta la elaboración de los "Pu Kuzaw" de su preferencia, asumiendo también su protagonismo en las funciones asignadas en los ceremoniales y en las actividades sociales que le encomiendan las autoridades comunitarias.

- Desarrollar el "Az Mapu" reconociendo inicialmente en ejemplos cotidianos de la vivencia familiar y comunitaria de valores ancestrales mapuche; tales como el "Ekvwvn", "Yamvwvn", la "Reciprocidad", la "Vida equilibrada y armoniosa" (tanto con la naturaleza como con la sociedad mapuce) y hasta explicar con precisión las manifestaciones del ser mapuche, de acuerdo al perfil que lo constituye: "Kim-ce" (persona con sabiduría); "Nor-ce" (persona correcta y respetuosa); "Kum-ce" (persona de buen corazón, generosa y solidaria); "Newen-ce" (persona equilibrada, sana y fuerte); "Shakin-ce" (persona sensible y alegre); y "Kvlfvn-ce" (persona servicial y ágil.

En el desarrollo de estos principios en la familia, en la Escuela y en la vida comunitaria, se va generando una práctica formativa y cultural Mapuche que se reproduce en cualquier relación de aprendizaje comunitario, entre otras "construir colaborativamente Planes y Programas de Estudios propios". Según esta precisión el aprendizaje en la cultura mapuche es siempre comunitario e interactivo. Sin duda que esta práctica es un factor que contribuyó a instalar con mucha facilidad el trabajo comunitario de construir Curriculum Crítico Emergente.

\section{2.- La Vivencia de la Comunidad de Aprendizaje en la Construcción Curricular Crítica Emergente de Llaguepulli.}

"Hemos aprendido con ustedes que los conocimientos o KIMUN Mapuche son siempre integrales y holísticos, y ustedes han aprendido con nosotros que el saber construir curriculum crítico emergente es también un conocimiento y un saber hacer integral y holístico. Coincidimos en la misma visión, por tanto, nuestra construcción seguirá en la visión Mapuche del aprendizaje colaborativo, interactivo y eminentemente comunitario" (Rolando Pinto, coordinador Académico del Proyecto de Construcción del Curriculum Crítico Emergente para la Escuela Básica Comunitaria KOM PU LOF ÑI KIMELTUWE de Llaguepulli, Taller de Elaboración de la Matriz Curricular, Agosto de 2011)

Enfrentados a la elaboración del primer instrumento técnico-pedagógico del Proyecto, resultaba potenciador para la construcción hacer visible la disposición colaborativa y la complementariedad de saberes y prácticas que debíamos instalar con la elaboración de esa 
Matriz Curricular. Y fue justamente esa actitud lo que generó la confianza y la legitimidad entre todos/as los/as miembros de ese equipo que siguió coordinando y orientando todas las acciones del Proyecto. Concebir ese trabajo de construcción curricular como una comunidad de aprendizaje interactivo y complementario entre directivos/autoridades y comunidad Mapuche Lafquenche y académicos/as chilenos, provenientes de varias Universidades pero todos/as con una visión humanista emancipadora, fue sin duda otro acierto de esta construcción curricular.

\section{3.- La Explicitación Ideológica Política de Entender la Construcción Curricular como un Artefacto Cultural Descolonizador.}

“La Investigación Acción Participativa no provoca una integración efectiva entre investigador y sector humano que aporta la información necesaria para la acción que se quiere desarrollar. El conocimiento técnico-instrumental sigue perteneciendo al investigador y el otro sólo sigue instrucciones u orientaciones del que sabe que es lo que se quiere investigar y actuar. Nosotros en la Araucanía hemos implementado una estrategia interactiva aplicada, donde la investigación y la acción la realiza un equipo integrado de Mapuche y académicos y conjuntamente vamos generando la información o el conocimiento necesario para actuar" (conversación de la Antropóloga Teresa Durán, de la Universidad Católica de Temuco, cuando le mostramos, en Marzo de 2012, la Matriz Curricular que habíamos generado interactivamente en Llaguepulli).

Esta conversación y la lectura de algunos textos metodológicos de autoría de Teresa Durán, nos permitió reorientar el trabajo de elaboración de cada Plan y Programa de Estudios en los ámbitos de saberes y prácticas ancestrales Mapuche Lafquenche, en una perspectiva descolonizadora y emancipativa. Se trataba de entender la reivindicación de soberanía cultural mapuche como una orientación vital; y para el equipo técnico académico, una posibilidad epistemológica de construir una acción curricular emancipadora. Para asegurar esta orientación, siempre al interior del equipo coordinador, el responsable del trabajo era el Director de la Escuela y el Logko de la Comunidad de Llaguepulli; los académicos sólo apoyábamos las decisiones sobre las acciones planificadas, pero siempre en una relación horizontal de respeto y equilibrio intercultural. Se creaba comunitaria e interactivamente, un aprendizaje social de liberación y de transformación.

Pues bien, son estos tres factores los que, de manera natural, se diera una perspectiva descolonizadora en la Construcción del Curriculum Crítico Emergente, para la Escuela Básica Comunitaria KOM PU LOF ÑI KIMELTUWE de Llaguepulli, Región de La Araucanía; esto mismo hace de esta construcción, una experiencia emancipadora de la pedagogía y el curriculum, innovadora e inédita en Chile.

Santiago, 16 de Enero de 2020. 


\section{BIBLIOGRAFÍA}

Durán, T. y otros. (2014). Antropología desde el Sur. Obras Escogidas. Ediciones Universidad Católica de Temuco. Colección Cátedra Fray Bartolomé de las Casas, Temuco, Chile.

Dussel, E. (2012). Para una Ética de la Liberación Latinoamericana. Editorial Didáctica, 03 Volúmenes, Buenos Aires, Argentina.

Dussel, E. (1974). Método para una Filosofía de la Liberación, Editorial Salamanca, España.

Escuela Básica Comunitaria KOM PU LOF ÑI KIMELTUWE (2016). Planes y Programas de Estudios propios de la Escuela Básica Particular Subvencionada "Kom Pu Lof Ñi Kimeltuwe". 05 volúmenes, Edición Digital, presentados al Consejo Regional de Educación de La Araucanía. Temuco, Chile.

Escuela Básica Comunitaria KOM PU LOF ÑI KIMELTUWE (2015). Proyecto Educativo Institucional. Edición Digital; Llaguepulli, Comuna de Teodoro Schmidt, Región de la Araucanía, Chile.

Freire, P. (1997). Pedagogía de la Autonomía. Siglo XXI Ediciones, México.

Pairican Padilla, F. (2019). "La disputa por las identidades bajo la comunidad imaginada chilena" En: Le Monde Diplomatique, Edición Chilena, Año XX, Número 211, Octubre 2019; pp. 6 y 7.

Pairican Padilla, F. (2014), Malon la Rebelión del movimiento Mapuche. Pehuén Editores, Santiago (Chile).

Pinto Contreras, R. (2008). El Currículo Crítico. Una pedagogía transformativa para la educación latinoamericana. Ediciones Universidad Católica de Chile, Colección Lecciones. Santiago, Chile.

Pinto Contreras, R.; Calfuqueo, Jorge, Painequeo, Hugo; Alejandra Silva M.; Froilán Cubillos A. y Roberto Berrios (2011). Proyecto UNESCO/IESALC/UMCE №82, Chile. Diseño de la Matriz Curricular. Escuela Básica KOM PU LOF ÑI KIMELTUWE, de Llaguepulli. Santiago, Noviembre de 2011.

Pinto Contreras, R. y Jorge Osorio Vargas (2014). Pedagogía Critica para una Educación Pública y transformativa en América Latina. Derrama Magisterial Ediciones, Colección Cooperación con la Formación Docente en América Latina. Lima, Perú.

Pinto Contreras, R. (2018). Educación entre Adultos. El Adulto como protagonista de su formación. Editorial UPLA, Valparaíso, Chile.

Pinto Contreras, R. (2018). Cosmovisiones Populares como fundamentos polisémicos de la Pedagogía Crítica. En proceso de edición. Santiago, Chile. 
Saavedra, A. (2006). Las transformaciones de la población Mapuche en el siglo XX. Ediciones GIA, Universidad Academia de Humanismo Cristiano, Santiago, Chile.

Samaniego, A. y Ruiz, C. (2007). Mentalidades y políticas wingka: pueblo Mapuche entre golpe y golpe. Colección América, Ediciones CSIC, USACH, Santiago (Chile). 